\title{
Low Power and High Fault Coverage BIST TPG
}

\author{
K.Vasudevareddy ${ }^{1}$ K.V.Ramanaiah ${ }^{2}$ K.Sravani ${ }^{3}$ \\ Asst.Proff NBKRIST Vidyanagar \\ Associate Professor YVU Proddatur \\ Asst.Proff NBKRIST Vidyanagar
}

\begin{abstract}
This paper presents a low hardware overhead test pattern generator (TPG) for scan-based Built-In Self-Test (BIST) that can reduce switching activity in circuits under test (CUTs) during BIST and also achieve very high fault coverage with reasonable lengths of test sequences. The proposed BIST TPG decreases transitions that occur at scan inputs during scan shift operations and hence reduces switching activity in the CUT. The proposed BIST is comprised of two TPGs: LT-RTPG and 3-weight WRBIST. Test patterns generated by the LT-RTPG detect easy-to-detect faults and test patterns generated by the 3-weight WRBIST detect faults that remain undetected after LT-RTPG patterns are applied. The proposed BIST TPG does not require modification of mission logics, which can lead to performance degradation. Experimental results for ISCAS'85 benchmark circuits demonstrate that the proposed BIST can significantly reduce switching activity during BIST while achieving $100 \%$ fault coverage for all ISCAS'85 benchmark circuits. Larger reduction in switching activity is achieved in large circuits. Experimental results also show that the proposed BIST can be implemented with low area overhead.
\end{abstract}

IndexTerms: Built-in self-test (BIST), heat dissipation during test application, low power testing, power dissipation during test application, random pattern testing.

\section{INTRODUCTION}

SINCE in built-in self-test (BIST), test patterns are generatedand applied to the circuit-under-test (CUT) by on-chiphardware, minimizing hardware overhead is a major concernof BIST implementation. Unlike stored pattern BIST, whichrequires high hardware overhead due to memory devices requiredto store precomputed test patterns, pseudorandom BIST, where test patterns are generated by pseudorandom patterngenerators such as linear feedback shift registers (LFSRs) andcellular automata (CA), requires very little hardware overhead.However, achieving high fault coverage for CUTs that containmany random pattern resistant faults (RPRFs) only with(pseudo) random patterns generated by an LFSR or CA oftenrequires unacceptably long test sequences thereby resulting inprohibitively long test time. The random pattern test lengthrequired to achieve high fault coverage is often determined by only a few RPRFs. Several techniques have been proposed to address thisproblem. Reseedable and/or reconfigurable LFSRs are random patterns that do notdetect any new faults are mapped into deterministic tests forRPRFs. In test point insertion (TPI) techniques, controland observation points are inserted at selected gates to improvedetection probabilities of RPRFs. In weighted random pattern ,testing the outputs of test pattern generator (TPG)are biased to generate test sequences that have nonuniformsignal probabilities to increase detection probabilities of RPRFsthat escape pseudorandom test sequences, which have a uniformsignal probability of 0.5 . Random pattern generatorsuse Markov sources to exploit spatialcorrelation between state inputs that are consecutively locatedin the scan chain. A 3-weight weighted random BIST (3-weightWRBIST) can be classified as an extreme case of conventionalweighted random pattern testing BIST. However, in contrastto conventional weighted random pattern testing BIST wherevarious weights, e.g., $0,0.25,0.5,0.75,1.0$, can be assignedto outputs of TPGs, in 3-weight WRBIST, only three weights, $0,0.5$, and 1 , are assigned. Since only three weights are used,circuitry to generate weights is simple; weight $1(0)$ is obtainedby fixing a signal to a $1(0)$ and weight 0.5 by driving a signalby an output of a pseudorandom pattern generator, such as anLFSR. Weight sets are calculated from test cubes for RPRFs.Though the attainment of high fault coverage with practicallengths of test sequences is still one major concern of BISTtechniques, reducing switching activity has become another importantobjective. It has been observed that switching activityduring test application is often significantly higher than thatduring normal operation .The correlation between consecutiverandom patterns generated by an LFSR is low-this is awell-known property of LFSR generated patterns. Onthe other hand, significant correlation exists between consecutivepatterns during the normal operation of a circuit. Hence,switching activity in a circuit can be significantly higher duringBIST than that during its normal operation. Finite-state machinesare often implemented in such a manner that vectorsrepresenting successive states are highly correlated to reduce power dissipation . However, use of designfor-testability(DFT) techniques such as scan significantly decreases the correlationbetween consecutive state 
vectors. Use of scan allows toapply patterns that cannot appear during normal operation to thestate inputs of the CUT during test application. Furthermore, thevalues applied at the state inputs of the CUT during scan shiftoperations represent shifted values of test vectors and circuit responsesand have no particular temporal correlation. Excessiveswitching activity due to low correlation between consecutivetest patterns can cause several problems. Since heat dissipation in a CMOS circuit is proportional toswitching activity, a CUT can be permanently damaged dueto excessive heat dissipation if switching activity in the circuitduring test application is much higher than that during its normaloperation. Heat dissipated during test application is already influencingthe design of test methodologies for practical circuits.

\section{A. GENERATOR}

\section{3-WEIGHT WRBIST}

In this paper, we assume that the Combinational CUT has primary and state inputs, and employs fullscan. Even though the proposed BIST TPG is applicable to scan designs with multiple scan chains, we assume that all primary and state inputs are driven by a single scan chain unless stated otherwise (application to multiple scan chains is discussed separately) only for clarity and convenience of illustration. A test cube is a test pattern that has unspecified inputs. The detection probability of a fault is defined as the probability that a randomly generated test pattern detects the fault. In the 3-weight WRBIST scheme, fault coverage for a random pattern resistant circuit is enhanced by improving detection probabilities of RPRFs; the detection probability of an RPRF is improved by fixing some inputs of the CUT to the values specified in a deterministic test cube for the RPRF.

\section{B. MINIMIZING SWITCHING ACTIVITY DURING BIST}

The BIST TPG proposed in this paper reduces switching activity in the CUT during BIST by reducing the number of transitions at scan inputs during scan shift cycles. In this paper, we assume that the sequential CUT is implemented in CMOS, and employs full-scan. If scan input $p_{i}$ is assigned $v$, where $v \in\{0,1\}$, at time $t-1$ and assigned the opposite value at time $t$; then a transition occurs at $p_{i}$ at time $t$; . The transition that occurs at scan input $p_{i}$ can propagate into internal circuit lines causing more transitions. During scan shift cycles, the response to the previous scan test pattern is also scanned out of the scan chain. Hence, transitions at scan inputs can be caused by both test patterns and responses. Since it is very difficult to generate test patterns by a random pattern generator that cause minimal number of transitions while they are scanned into the scan chain and whose responses also cause minimal number of transitions while they are scanned out of the scan chain, we focus on minimizing the number of transitions caused only by test patterns that are scanned in. Even though we focus on minimizing the number of transitions caused only by test patterns, our extensive experiments show that the proposed TPG can still reduce switching activity significantly during BIST .Since circuit responses typically have higher correlation among neighbourhood scan outputs than test patterns, responses cause fewer transitions than test patterns while being scanned out.

A transition at the input of the scan chain at scan shift cycle $t$, which is caused by scanning in a value that is opposite to the value that was scanned in at the previous scan shift cycle $t-1$, continuously causes transitions at scan inputs while the value travels through the scan chain for the following scan shift cycles. Fig. 5 describes scanning a scan test pattern 01100 into a scan chain that has five scan flip-flops. Since a 0 is scanned into the scan chain at time $t ;=0$, the 1 that is scanned into the scan chain at time $t ;=1$ causes a transition at the input of the scan chain and continuously causes transitions at the scan flip-flops it passes through until it arrives at its final destination $p_{i}$ at time $t,=5$. In contrast, the 1 that is scanned into the scan chain at the next cycle $t:=2$ causes no transition at the input of the scan

chain and arrives at its final destination $p_{2}$ at time $t:=5$ without causing any transition at the scan flipflops it passes through. This shows that transitions that occur in the entire scan chain can be reduced by reducing transitions at the input of the scan chain. Since transitions at scan inputs propagate into internal circuit lines causing more transitions, reducing transitions at the input of scan chain can eventually reduce switching activity in the entire circuit.

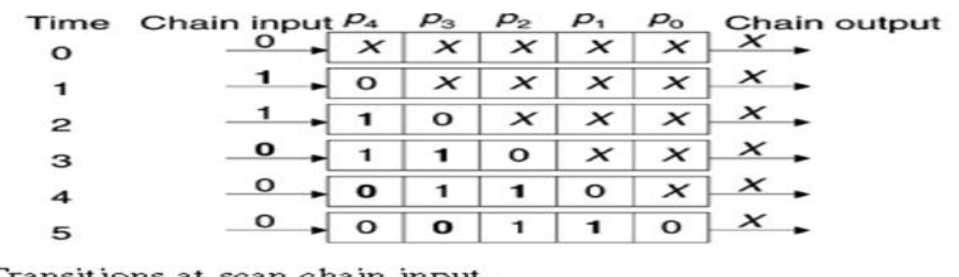

Fig. 5. Transitions at scan chain input. 


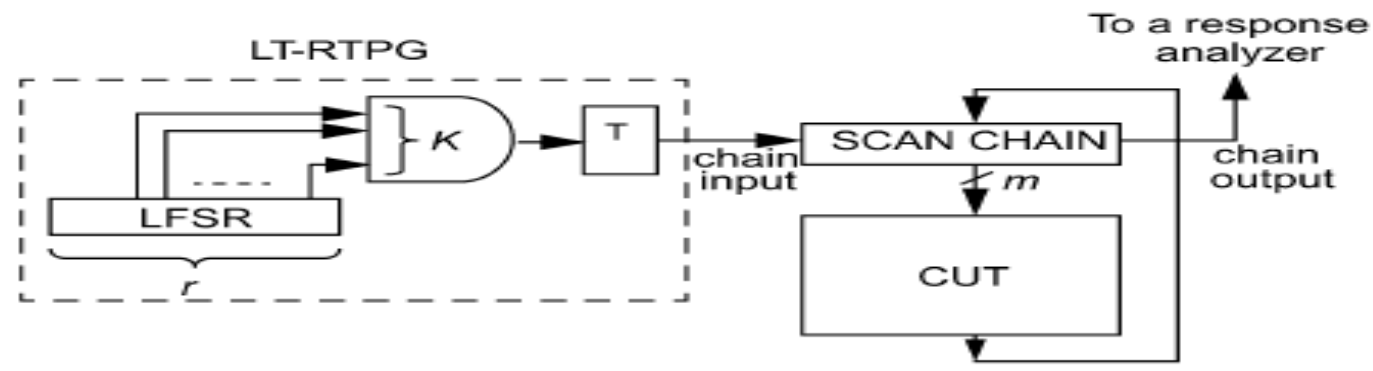

Fig. 6. LT-RTPG.

In 3-Weighted Random Built-In-Self Test, the combinational CUT has 'm' primary and state inputs, and employs full-scan. Even though the implemented BIST TPG is applicable to scan designs with multiple scan chains, the all primary and state inputs are driven by a single scan chain unless stated otherwise (application to multiple scan chains ) only for clarity and convenience of illustration. A test cubeis a test pattern that has unspecified inputs. The detection probabilityof a fault is defined as the probability that a randomly generated test pattern detects the fault. In the 3-weight WRBIST scheme, fault coverage for a random pattern resistant circuit is enhanced by improving detection probabilities of RPRFs, the detection probability of an RPRF is improved by fixing some inputs of the CUT to the values specified in a deterministic test cube for the RPRF. A generator or weight setis a vector that represents weights that are assigned to inputs of the circuit during 3-weight WRBIST. Inputs that are assigned weight 1(0) are fixed to 1(0) and inputs that are assigned weight 0.5 are driven by outputs of the pseudorandom pattern generator, such as an LFSR and a CA. A generator is calculated from a set of deterministic test cubes for RPRF's.

Pseudo-random pattern sequences generated by an LFSR and a CA are modified (fixed) by controlling the AND and OR gates with overriding signals S0 and S1, fixing a random value to a ' 0 ' is achieved by setting ' $\mathrm{S} 0$ ' to a ' 1 ' and ' $\mathrm{S} 1$ ' to a' 0 ' and fixing a random value to a ' 1 ' is achieved by setting ' $\mathrm{S} 1$ ' to a ' 1 '. Overriding signals 'S0' and 'S1' are driven by the outputs of T flip-flops, TF0 and TF1 .The inputs of TF0 and TF1 are in turn driven by the outputs of the decoding logic 'D0' and 'D1' respectively, which are generated by the outputs of the shift counter and the generator counter as inputs.

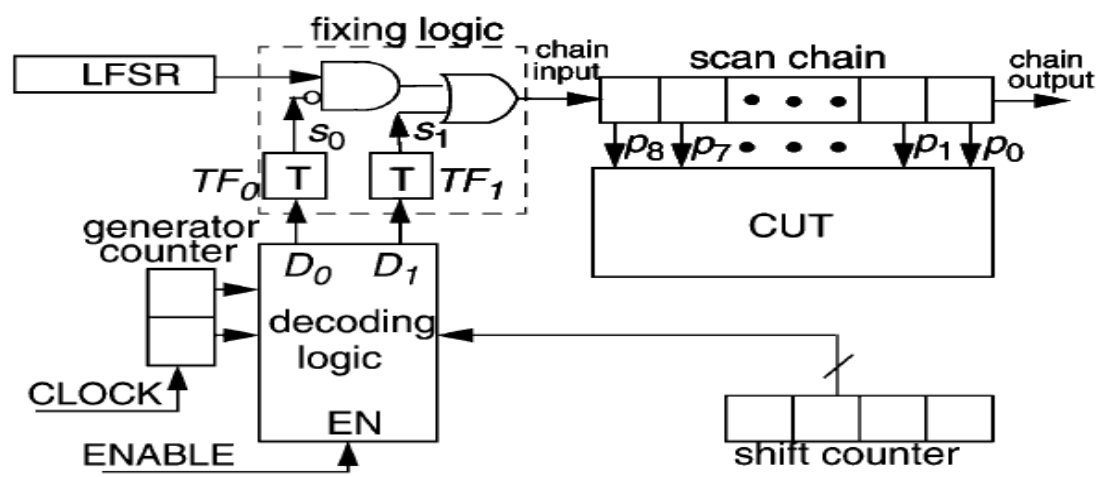

Figure 6: 3-Weight Weighted Random BIST

Hence, hardware overhead for implementing a 3-weight WRBIST is incurred only by the decoding logic and the fixing logic, which includes two toggle flip-flops, an AND and an OR gate. Since the fixing logic can be implemented with very little hardware, overall hardware overhead for implementing the serial fixing 3weight WRBIST is determined by hardware overhead for the decoding logic.

\section{LT-RTPG USING BASIC LINEAR FEEDBACK SHIFT REGISTER (LFSR)}

The Low Transition Random Test Pattern Generator (LT-RTPG) reduces switching activity during BIST by reducing transitions at scan inputs during scan shift operation. The LT-RTPG is comprised of an rstage LFSR, a K-input AND gate, and a toggle flip-flop (T flip-flop). Hence, it can be implemented with very little hardware. Each of ' $\mathrm{K}$ ' inputs of the AND gate is connected to either a normal or an inverting output of the LFSR stages. If large $\mathrm{K}$ is used, large sets of neighboring state inputs will be assigned identical values in most test patterns, resulting in the decrease fault coverage or the increase in test sequence length. In this work, LTRTPGs with only $\mathrm{K}=2$ or 3 are used since a $\mathrm{T}$ - flip-flop holds previous values until the input of the $\mathrm{T}$ flip-flop is assigned a 1,the same value ' $\mathrm{v}$ ', where $\mathrm{v} \mathrm{E}\{0,1\}$, is repeatedly scanned into the scan chain until the value at the output of the AND gate. Hence, adjacent scan flip-flops are assigned identical values in most test patterns 
and scan inputs have fewer transitions during scan shift operations. Since most switching activity during scan BIST occurs during scan shift operations. The LT-RTPG can reduce heat dissipation during overall scan testing. LT-RTPG detects Easy-to- detect faults, those faults are left over by LT-RTPG, are called Random Pattern Resistant Faults (RPRF).

It has been observed that many faults that escape random patterns are highly correlated with each other and can be detected by continuously complementing values of a few inputs from a parent test vector. These observations are exploited and improve fault coverage for circuits that have large numbers of RPRFs. I have also observed that tests for faults that escape LT-RTPG test sequences share many common input assignments.

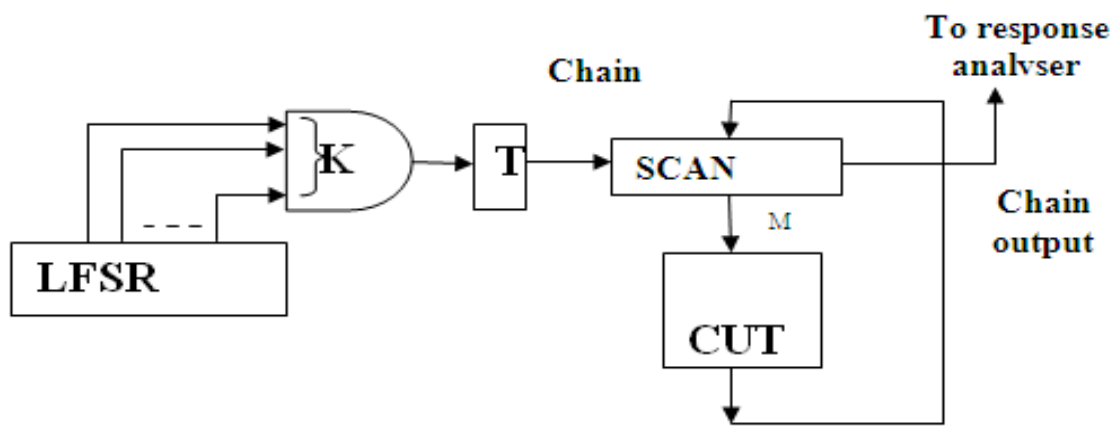

Figure 6.1: LT-RTPG using basic LFSR

This implies that RPRFs that escape LT-RTPG test sequences can be effectively detected by fixing selected inputs to binary values specified in deterministic test cubes for these RPRFs and applying random patterns to the rest of inputs. This technique is used in the 3-weight WRBIST to achieve high fault coverage for random pattern resistant circuits.

\section{PROPOSED TEST PATTERN GENERATOR}

The proposed BIST is comprised of two TPGs: an LT-RTPG and a 3-weight WRBIST (see Fig. 7). The multiplexer, which drives the input of scan chain, selects a test pattern source between the LT-RTPG and the 3weight WRBIST.

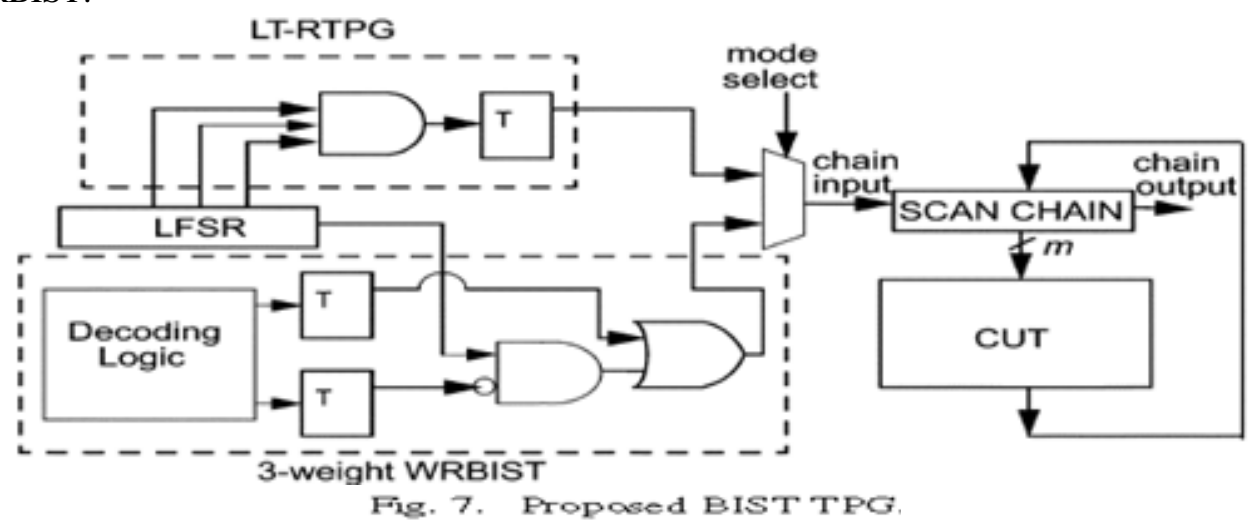

In the first test session, test patterns generated by the LT-RTPG are selected and scanned into the scan chain to detect easy-to-detect faults. In the second session, test patterns that are generated by the 3-weight WRBIST are selected to detect the faults that remain undetected after the first session. Considering the fact that an LT-RTPG can be implemented with very little hardware overhead (only one flip-flop and one AND gate in addition to an LFSR), overall hardware overhead to implement the proposed TPG is determined by hardware overhead for the decoding logicof the 3-weight WRBIST.

\subsection{Test pattern of $\mathrm{C} 17$ circuit:}

\section{SIMULATION RESULTS:}

In the below simulation window, Fig $7.3 \mathrm{C} 17$ circuit predefined test pattern output, The clock frequency is set to $10 \mathrm{MHz}$.In order get random pattern resistant faults I need to have predefined test patterns of faulty $\mathrm{C} 17$ circuit. Normally 4 faults have been inserted into $\mathrm{C} 17$ circuit. The different test patterns generated by the LFSR are applied to C17 circuit then at each and every fault corresponding test vector is noted. These patterns are the pre-defined test vectors of $\mathrm{C} 17$ circuit. 

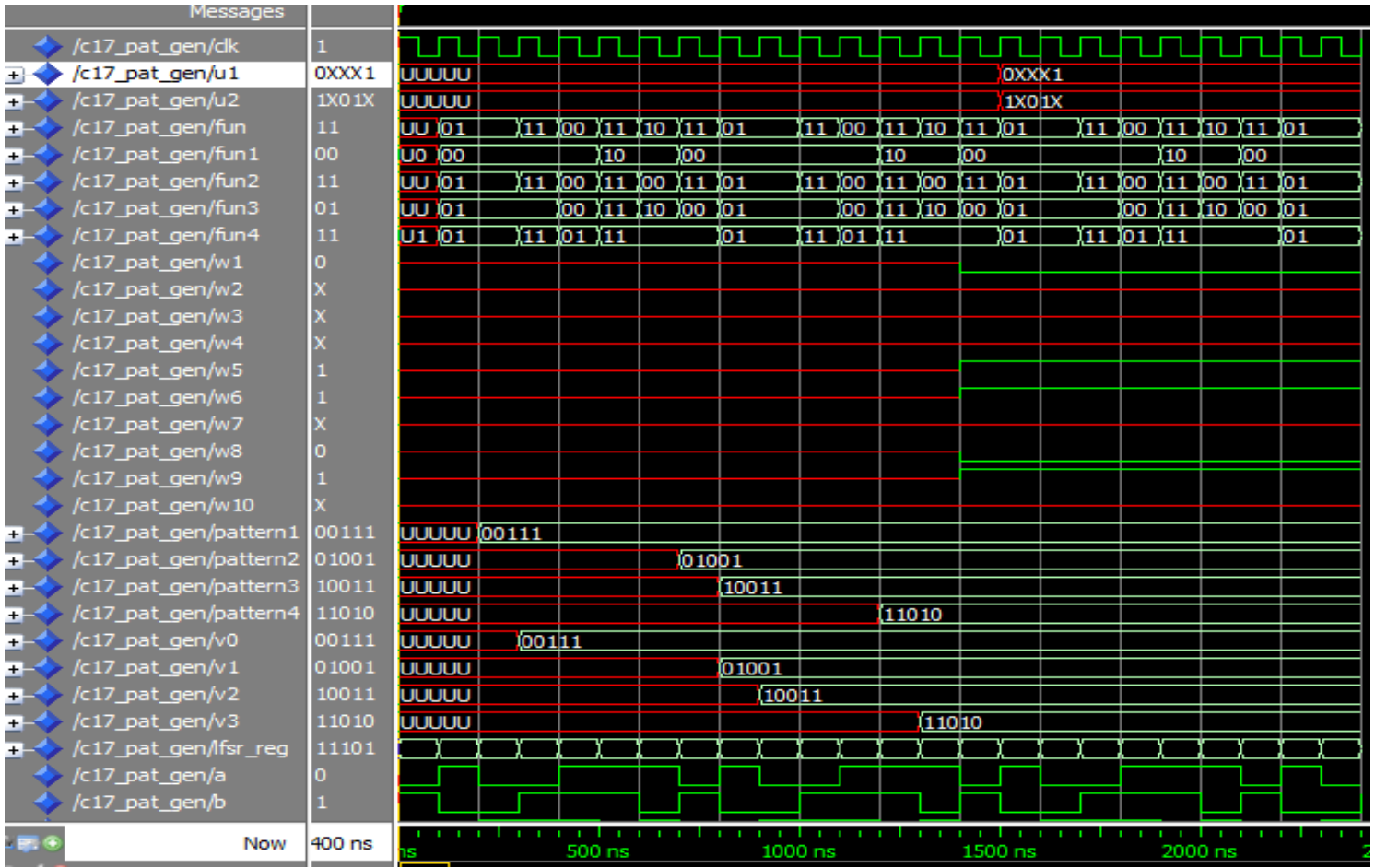

Figure 8.1: C17 circuit predefined test pattern output

\subsection{Simulated output of Implemented system}

In the below simulation window, Fig 7.4 Complete Implemented system output, the clock frequency is set to 10MHz.Both easy to detect faults and random pattern resistant faults are detected .The fault signal is high ,the value of fault covered as 9 . Hence the fault coverage is improved to $25 \%$.

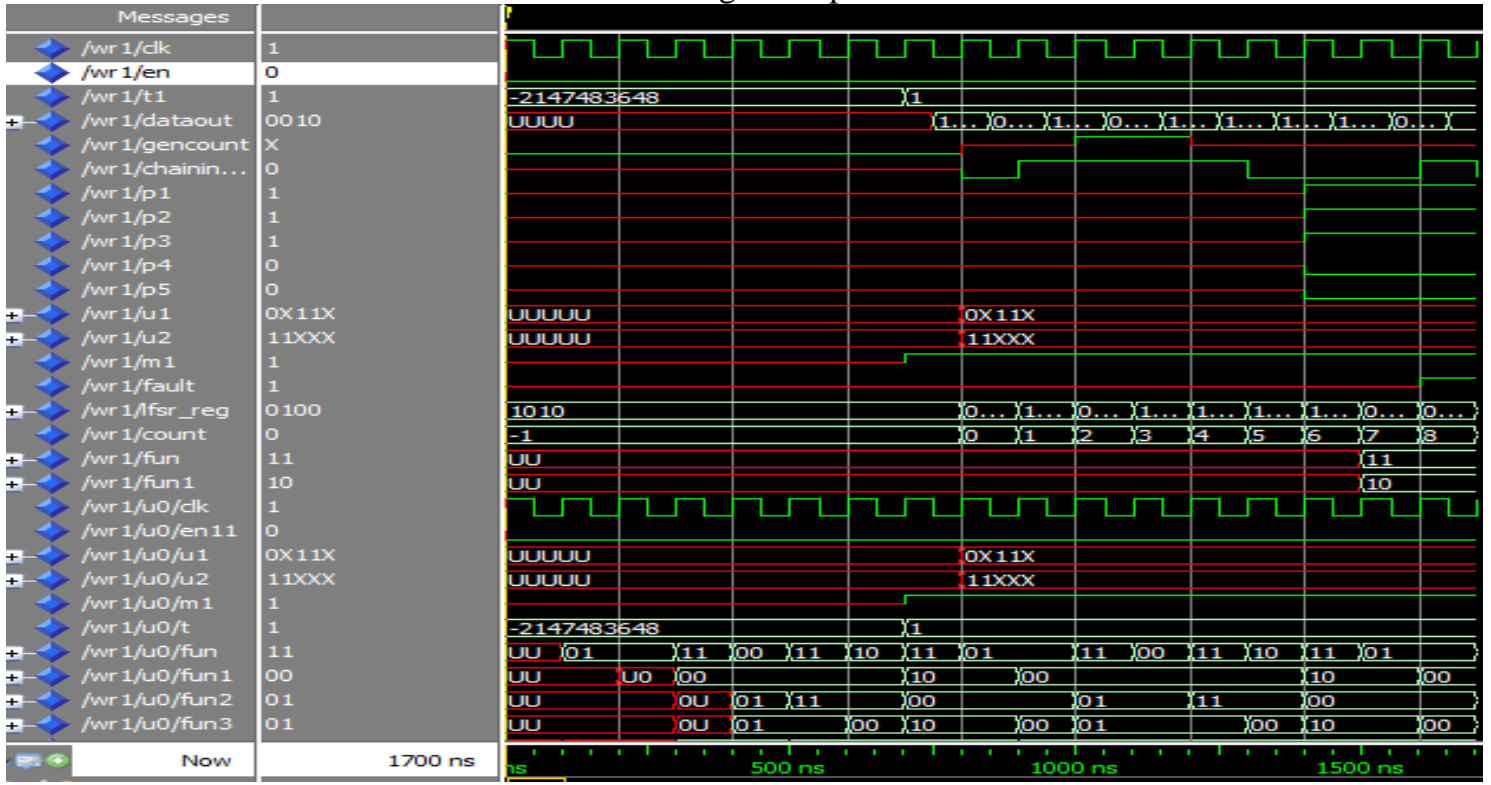

Figure 8.2: Proposed system output

This window consist of LFSR generated patterns which will be labeled as lfsr_reg. After applying C17 circuit predefined patterns 1000,1001 as set, reset values the final fault becomes high ,for 0000,1111 values final fault signal is low. Hence the fault final signal will be high only for the predefined test patterns of $\mathrm{C} 17$ circuit

\section{SYNTHESIS RESULTS}

\subsection{Gate count for implemented system}

The below figure shows gate count report for implemented system, Total equivalent gate count for the design is 324.Additional JTAG gate count for IOBs is 912 gates. 


\begin{tabular}{|c|c|c|c|}
\hline \multicolumn{4}{|c|}{ ZZ Project Status } \\
\hline Project File: & zz.ise & Current State: & Placed and Routed \\
\hline Module Name: & exs_cc & • Errors: & No Errors \\
\hline Target Device: & $x c 2 s 600 e-\pi g 456$ & -Warnings: & 12 Wamings \\
\hline Product Version: & ISE. 8.1i & - Updated: & Fri Jan 4 00:45:19 2013 \\
\hline
\end{tabular}

\begin{tabular}{|c|c|c|c|c|}
\hline \multicolumn{5}{|c|}{ Device Utilization Summary } \\
\hline Logic Utilization & Used & Available & Utilization & Note(s) \\
\hline Number of Slice Flip Flops & 14 & 13,824 & $1 \%$ & \\
\hline Number of 4 input LUTs & 34 & 13.824 & $1 \%$ & \\
\hline \multicolumn{5}{|l|}{ Logic Distribution } \\
\hline Number of occupied Slices & 22 & 6.912 & $1 \%$ & \\
\hline Number of Slices containing only related logic & 22 & 22 & $100 \%$ & \\
\hline Number of Slices containing unrelated logic & 0 & 22 & $0 \%$ & \\
\hline Total Number of 4 input LUTs & 34 & 13.824 & $1 \%$ & \\
\hline Number of bonded $\underline{10 B s}$ & 18 & 325 & $5 \%$ & \\
\hline IOB Flip Flops & 1 & & & \\
\hline Number of GCLKs & 1 & 4 & $25 \%$ & \\
\hline Number of GCLKIOBs & 1 & 4 & $25 \%$ & \\
\hline Total equivalent gate count for design & 324 & & & \\
\hline Additional JTAG gate count for IOBs & 912 & & & \\
\hline
\end{tabular}

Figure 7.3: Proposed system synthesis report

7.4.2 Usage of power, voltage and current:

\begin{tabular}{|c|c|c|}
\hline Power summary: & $(\mathbf{m A})$ & $\#(\mathbf{m W}$ \\
\hline Total estimated power consumption: & & 936 \\
\hline Vecint 2.50V: & 372 & 929 \\
\hline Veco33 3.30V: & 2 & 7 \\
\hline Clocks: & 331 & 826 \\
\hline Inputs: & 40 & 100 \\
\hline Logic: & $\mathbf{0}$ & $\mathbf{0}$ \\
\hline \multicolumn{3}{|l|}{ Outputs: } \\
\hline Vcco33 & $\mathbf{0}$ & $\mathbf{0}$ \\
\hline Signals: & $\mathbf{0}$ & $\mathbf{0}$ \\
\hline Quiescent Vccint 2.50V: & $\mathbf{1}$ & 3 \\
\hline Quiescent Vcco33 3.30V: & 2 & 7 \\
\hline \multicolumn{3}{|l|}{ Thermal summary: } \\
\hline Estimated junction temperature: & & $57 \mathrm{C}$ \\
\hline Ambient temp: & & $25 \mathrm{C}$ \\
\hline Case temp: & & $54 \mathrm{C}$ \\
\hline Theta J-A: & & $34 \mathrm{CW}$ \\
\hline
\end{tabular}

Figure 7.4: Power consumption analysis report

\section{CONCLUSION}

This paper presents a low hardware overhead TPG for scan based BIST that can reduce switching activity in cuts during BIST and also achieve very high fault coverage with a reasonable length of test sequence. The main objective of most recent BIST techniques has been the design of TPGs that achieve high fault coverage at acceptable test lengths for such circuits. While this objective still remains important, reducing heat dissipation during test application is also becoming an important objective. Since the correlation between consecutive patterns applied to a circuit during BIST is significantly lower, switching activity in the circuit can be significantly higher during BIST than that during its normal operation. Excessive switching activity during test application can cause several problems. The proposed TPG reduces the number of transitions that occur at scan inputs during scan shifting by scanning in the test patterns where neighbouring bits are highly correlated. The proposed BIST is comprised of two TPGs: LT-RTPG and 3-Weight WRBIST. Test sequences generated by the LT-RTPG detect easy-to-detect faults. Faults that escape LT-RTPG test sequences are detected by test patterns generated by the 3-Weight WRBIST.Hardware overhead for the proposed TPG is further reduced by identifying compatible scan chains in multiple scan chain designs.

Experimental results for ISCAS'85 benchmark circuits demonstrate that the proposed BIST can significantly reduce switching activity during BIST while achieving $100 \%$ fault coverage for all benchmark circuits. Larger reduction in switching activity is achieved for large circuits, which have long scan chains. The proposed BIST structure does not require modification of mission logic which can cause performance degradation. Experimental results for large industrial circuits demonstrate that the proposed TPG can significantly improve fault coverage of LFSR generated test sequences with low hardware overhead. 


\section{REFERENCES:}

[1]. Parag K.Lala“'An Introduction to Logic Circuit Testing”, Morgan and Clay Pool Publishers.

[2]. Rolf Isermann," Fault-Diagnosis Systems- An Introduction from Fault Detection to Fault Tolerance".

[3]. Muhammad M.Kellah,'Low Power Digital CMOS VLSI circuits and design methodologies".

[4]. H.-C. Tsai, K.-T. Cheng, C.-J. Lin, and S. Bhawmik,Low-Power VLSI Design-An Overview'

[5]. Sung-Mo Kang Yusuf Leblibici,"CMOS Digital Integrated Circuits Analysis and Design"-Third Edition.

[6]. B. Koenemann, "LFSR-Coded Test Patterns for Scan Design", Proc. of EuropeanTest Conference, Munich, Germany, April 1991,pp. 237-242.

[7]. N. Tamarapalli and J. Rajski, "Constructive multi-phase test point in-sertion for scan-based BIST," in Proc. IEEE Int. Test Conf., 1996, pp. 649-658.

[8]. Irith Pomeranz,"3-Weight Pseudo-Random Test Generation Based on a Deterministic Test Set for Combinational and Sequential Circuit, "in Proc IEEE vol,.12.no.7 July 2002.

[9]. Seongmoon Wang ,"A BIST TPG for Low PowerDissipation and High Fault Coverage",IEEE transactions on very large scale integration (VLSI) systems, vol. 15, no. 7, July 2007

[10]. S. Wang and S. K. Gupta, "LT-RTPG: A new test-per-scan BIST TPG for low heat dissipation," IEEE Trans. Comput.-Aided Des. Integer. CircuitsSyst., vol. 25, no. 8, pp. 1565-1574, Aug. 2006. 